\title{
Karyotype Evolution in the Horseshoe Bat Rhinolophus sedulus by Whole-Arm Reciprocal Translocation (WART)
}

\author{
Marianne Volleth $^{a}$ Klaus-Gerhard Heller ${ }^{b}$ Hoi-Sen Yong ${ }^{d}$ Stefan Müller ${ }^{c}$ \\ ${ }^{a}$ Department of Human Genetics, Otto von Guericke University, Magdeburg, ${ }^{b}$ Department of Biology, Humboldt \\ University, Berlin, and 'Institute of Human Genetics, University Hospital, Ludwig Maximilian University, Munich,

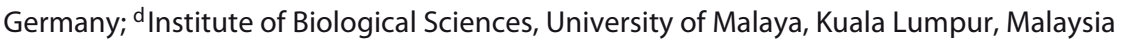

\section{Key Words}

Chiroptera · FISH · Karyotype evolution · Mammalia ·

Rhinolophidae · WART tence of a hybrid zone at the sampling locality is thought to be rather improbable, the WART may indicate ongoing karyotype evolution in this taxon.

(c) 2014 S. Karger AG, Basel

\begin{abstract}
Robertsonian (centric) fusion or fission is one of the predominant modes of chromosomal rearrangement in karyotype evolution among mammals. However, in karyotypes composed of only bi-armed chromosomes, creation of new chromosomal arm combinations in one step is possible only via whole-arm reciprocal translocation (WART). Although this type of rearrangement has often been proposed to play an important role in chromosomal evolution, direct observations of WARTs remained rare, and, in most cases, were found in hybrids of chromosomal races in the genera Mus and Sorex. For the first time, we present the karyotype of the horseshoe bat species Rhinolophus sedulus $(2 \mathrm{n}=28, \mathrm{FNa}=$ 52), where a WART between 2 metacentric autosomes was detected by G-banding and confirmed by FISH with painting probes of the vespertilionid bat Myotis myotis. Among the 6 specimens analyzed, 2 showed the heterozygous condition of the WART, 1 showed the presumed ancestral, and 3 specimens showed the derived homozygous state. As the exis-
\end{abstract}

Karyotypes may be shaped by different kinds of rearrangements, such as inversions, translocations, and Robertsonian (centric) fissions and fusions. The last type is thought to be the most common mode within Mammalia, at least among rearrangements detected by conventional cytogenetic techniques in the past. A relatively rarely reported type of rearrangement is whole-arm reciprocal translocation (WART) by which entire chromosomal arms are reciprocally exchanged between 2 chromosomes. In case that both chromosomes involved are (sub) metacentric, the term type-a WART was introduced [Hauffe and Pialek, 1997]. In type-b WARTs, one chromosome is subtelocentric and type-c WARTs require 3 chromosomes involved in the translocation.

Since the proposal of Winking [1986], WARTs have been postulated by several authors to be an important mode of rearrangement shaping especially karyotypes dominated by bi-armed chromosomes. Well-described

\section{KARGER}

E-Mail karger@karger.com www.karger.com $/ \mathrm{cgr}$
(C) 2014 S. Karger AG, Basel

$1424-8581 / 14 / 1434-0241 \$ 39.50 / 0$ 
examples are the chromosomal races of the common shrew in Scandinavia, which evolved through a series of WARTs with one exchange each between adjacent races [e.g. Halkka et al., 1987; Fredga, 1996]. In the order Rodentia, the potential of WARTs to rapidly create new chromosomal variants has been discussed for Mus m. domesticus [Hauffe and Pialek, 1997; Britton-Davidian et al., 2005] and the species complex of M. minutoides [Veyrunes et al., 2010].

In contact zones of chromosomal races, hybrids are detected due to the heterozygous condition for the metacentric chromosomes involved in the WART, i.e. they are heterozygotes with monobrachial homology [Narain and Fredga, 1996]. These hybrids represent events which occurred through a secondary contact and after the fixation of a WART in one of the populations involved. However, prior to fixation of a recently emerged WART in a population, specimens with monobrachial homology should also be observed. Such direct observation of a WART, i.e. the detection of a newly formed, recently evolved Robertsonian fusion chromosome, was only rarely reported. There are few examples in hybrid zones of chromosomal races in the house mouse [Capanna and Redi, 1995; Castiglia and Capanna, 1999; Catalan et al., 2000] as well as in the common shrew [Pavlova et al., 2008; Fedyk and Chetnicki, 2009]. Further, in 2 (related) specimens of $M$. minutoides and in a single individual of Tupaia glis, the spontaneous occurrence of a WART has been observed [Hirai et al., 2002; Veyrunes et al., 2007].

The evolutionary emergence of a WART would be initiated by a single specimen possessing 4 chromosomes with monobrachial homology, with the derived combination of chromosomal arms present in the heterozygous state. When the process of fixation is nearly completed, the population should mainly be composed of homozygotes for the new arm combination and only few specimens should retain the heterozygous condition. This stage is probably found in the agile gibbon Hylobates agilis in Sumatra [Hirai et al., 2005] and Thailand [Tanomtong et al., 2010]. Here, the ancestral arm combination, which is present in the closely related species $H$. albibarbis and H. muelleri, was found in the heterozygous, but never in a homozygous condition. In sum, WARTs have been proposed for taxa characterized by rapid chromosomal evolution which is evident by the presence of chromosomal races (Mus, Sorex) or a high number of rearrangements between closely related taxa (e.g. Hylobates).

The rate of chromosomal evolution in bats (i.e. the mammalian order Chiroptera) is generally thought to be slow [Baker and Bickham, 1980]. But among the 18 bat
Table 1. RSE specimens examined

\begin{tabular}{llrlll}
\hline ID & Sex & $\begin{array}{l}\text { SMF } \\
\text { No. }\end{array}$ & Condition & $\begin{array}{l}\text { WART } \\
\text { constitution }\end{array}$ & Remarks \\
\hline 207 & female & 69279 & homozygous & 5b, 5b, 6b, 6b \\
214 & male & 69280 & heterozygous & 5a, 5b, 6a, 6b & \\
325 & female & 87479 & heterozygous & 5a, 5b, 6a, 6b & mother of No. 326 \\
326 & female & - & homozygous & 5a, 5a, 6a, 6a & fetus of No. 325 \\
355 & female & 89140 & homozygous & 5b, 5b, 6b, 6b mother of No. 356 \\
356 & female & 89141 & homozygous & 5b, 5b, 6b, 6b juvenile of No. 355
\end{tabular}

ID = Identification number; SMF No. = accession number of the Senckenberg Museum, Frankfurt, Germany; WART constitution = constitution of the chromosomes involved in WART.

families with more than 1,100 species [Simmons, 2005], taxa with a rapid chromosomal evolution have also been found, especially in the family Phyllostomidae [Baker and Bickham, 1980]. The predominant mode of rearrangement in chiropteran karyotype evolution is Robertsonian translocation [Volleth and Eick, 2012]. This has also been proven to be true in the family Rhinolophidae [Mao et al., $2007,2008]$. As the number of common arm combinations in the Robertsonian fusion products revealed to be rather low, Mao et al. [2007, 2008] suggested that due to the prevalence of monobrachial homologies between taxa, WARTs have probably played an important role in the chromosomal evolution of this family.

The present study deals with the first karyotype description of the lesser woolly horseshoe bat, Rhinolophus sedulus (RSE), which is restricted to western Malaysia and Borneo without any described subspecies. In the study area, only 2 adults were found together in a day roost, partly with one young, thus probably forming monogamous pairs [Heller et al., 1993]. RSE presents the lowest diploid chromosome number found so far in the horseshoe bat family with a Y-autosome translocation and an autosomal type-a WART. The importance of this finding for karyotype evolution in the genus Rhinolophus will be discussed.

\section{Materials and Methods}

All 6 RSE specimens examined were caught at their day roosts in the vicinity of the Ulu Gombak Field Studies Centre of the University of Malaya $\left(3^{\circ} 20^{\prime} \mathrm{N}, 101^{\circ} 45^{\prime} \mathrm{E}\right)$ near Kuala Lumpur in the years 1984, 1989 and 1992. Accession numbers of the Senckenberg Museum, Frankfurt, Germany are given in table 1.

Chromosome preparations and FISH were carried out as described in Volleth et al. [2013]. For FISH, whole chromosome 


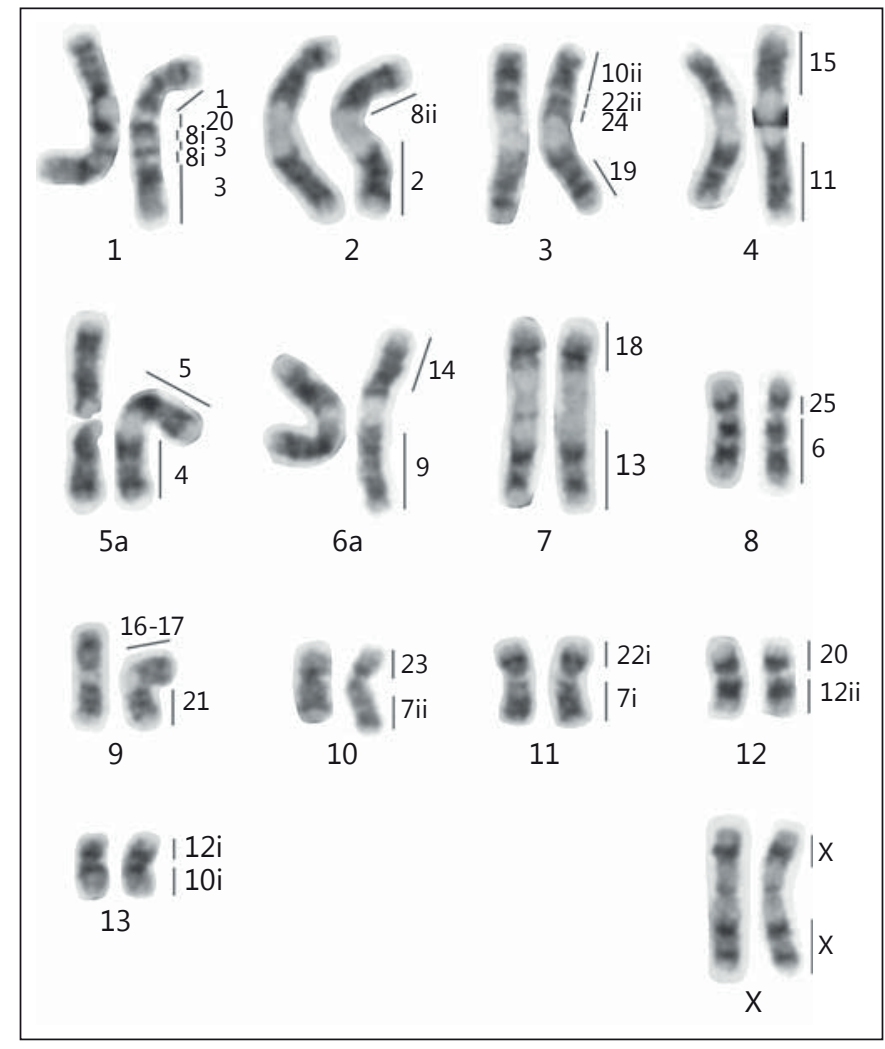

Fig. 1. G-banded karyotype of RSE female No. 326 showing the putatively ancestral homozygous condition for pairs 5 and 6 . Numbers to the right of each chromosome pair indicate homology to MMY chromosomes or chromosomal segments as revealed by FISH with MMY, EMA and TBE probes and G-band comparison. The appendix ' $i$ ' refers to the proximal, 'ii' to the distal part of the respective MMY chromosome (see also Materials and Methods). G-negative pericentromeric regions consist of heterochromatic material (see fig. 4).

painting probes from Myotis myotis (MMY) [Ao et al., 2006], T. belangeri [Müller et al., 1999] and Eulemur macaco [Müller et al., 1997] were used. Some Myotis probes painted 2 chromosome segments in RSE. In these cases, the proximal segment of the Myotis chromosome is indicated by ' $i$ ' and the distal segment by 'ii'.

\section{Results}

\section{Conventional Cytogenetics}

The karyotype of RSE shows a diploid chromosome number $(2 \mathrm{n})$ of 28 and a fundamental number of autosomal arms (FNa) of 52 . It consists of 7 large, 1 mediumsized and 5 small meta- to submetacentric autosomal pairs. The $\mathrm{X}$ chromosome is a large metacentric chromosome.

WART in Rhinolophus sedulus

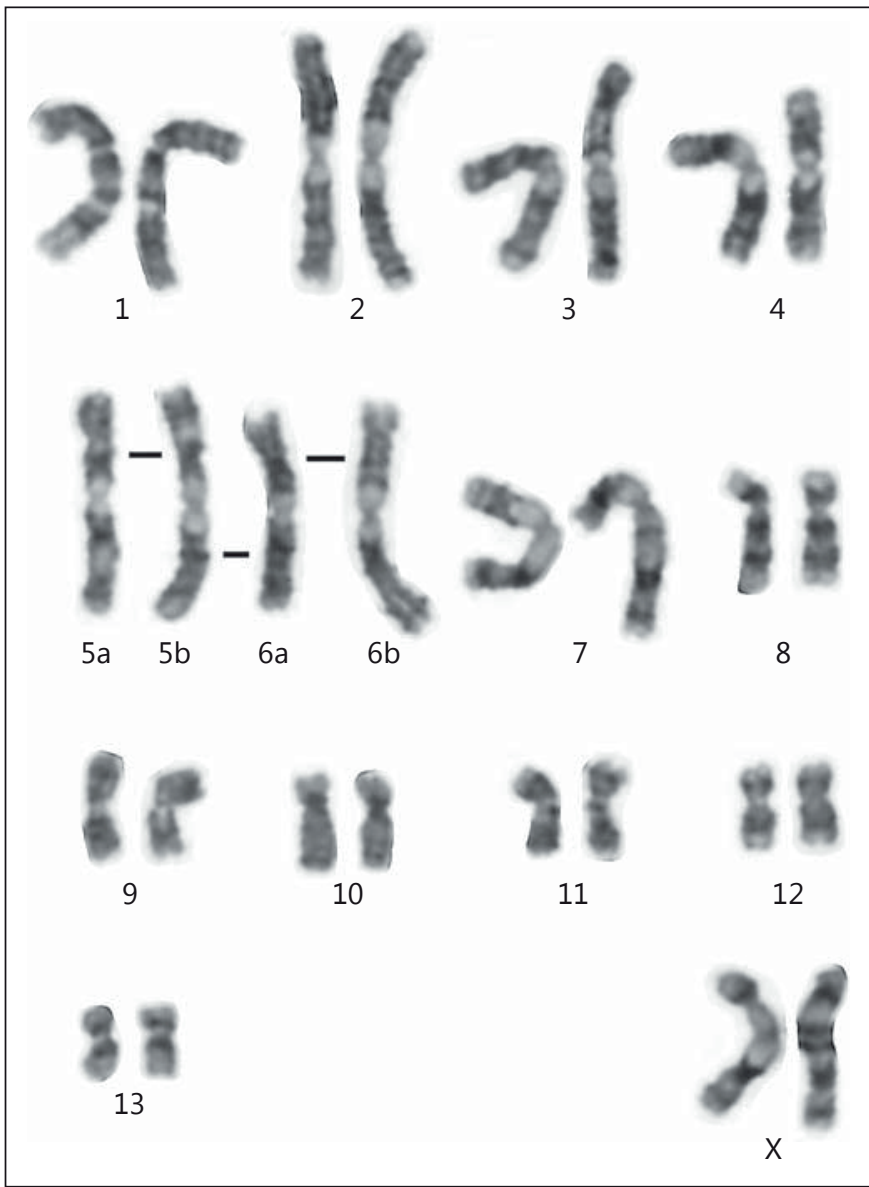

Fig. 2. G-banded karyotype of RSE female No. 325 showing the heterozygous condition for the WART. Dashes indicate homologous arms. The ancestral arm combination is present in RSE5a and RSE6 $a$ and the derived combination in RSE5b and RSE6b.

Comparative analysis showed that the banding pattern of autosomal pairs 1-4 and 7-13 was identical in all specimens studied. However, differences were noticed in pairs 5 and 6 concerning the arm composition. In figures 1 and 2, G-banded karyotypes of a RSE female (No. 325) and her fetus (No. 326) are presented. In contrast to the fetus (fig. 1), where the homologs of pairs 5 and 6 showed an identical banding pattern, in female No. 325, 4 chromosomes displayed only monobrachial homology (fig. 2). Two of these 4 chromosomes showed the same banding pattern as in the fetus. The remaining 2 chromosomes are the result of a WART between these pairs. In the following, the arm combination found in the chromosomal pairs of the RSE fetus will be indicated as RSE5a and RSE6a, respectively. A chromosome composed of the short arm of RSE5a and the long arm of RSE6a will be in- 
Fig. 3. G-band comparison of the 2 RSE pairs involved in the WART. The homozygous state shown on the left is presumed to be the ancestral condition because the arm combination MMY4/5 was also found in an out-group taxon. In the middle, the heterozygous state (No. 325) is shown, and on the right, the presumably derived homozygous condition (No. 356).

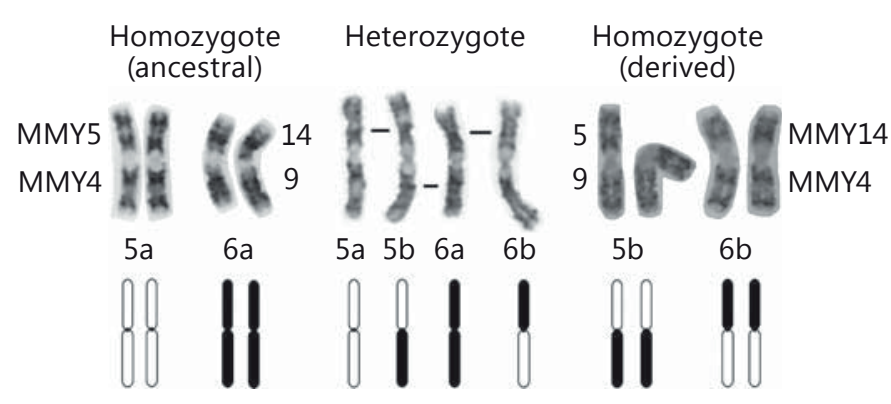

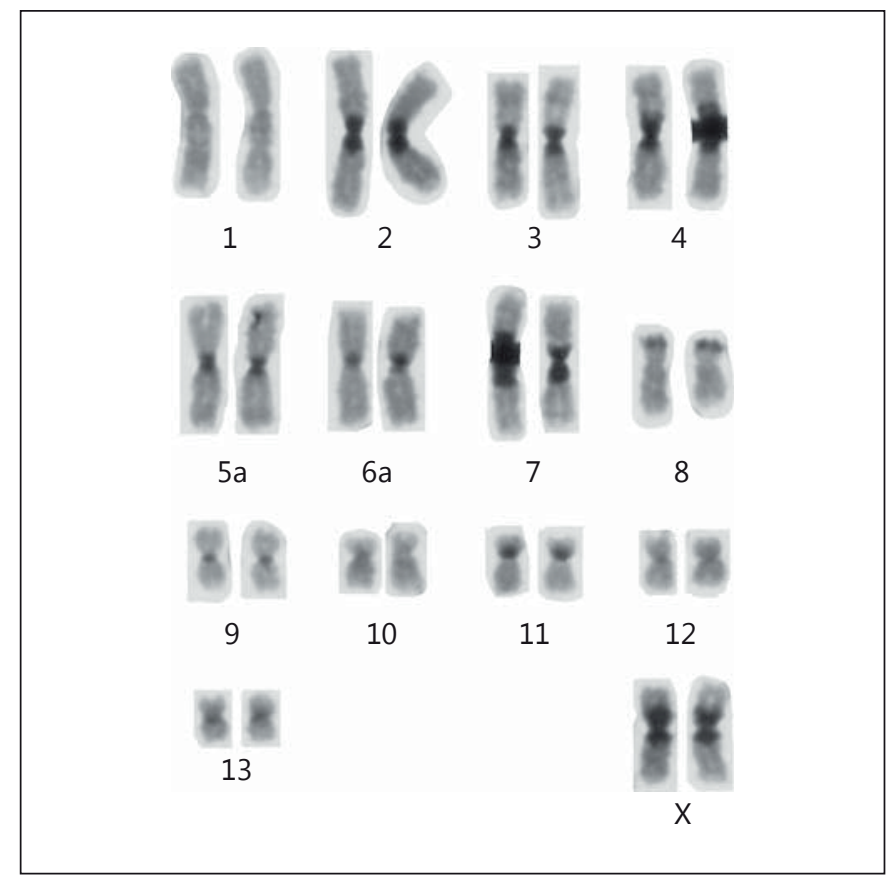

Fig. 4. C-banded karyogram of RSE female No. 326. The pairs were identified by QFQ banding prior to CBG staining. Please note the large pericentromeric blocks on pairs 2-7 and the $\mathrm{X}$ chromosome.

dicated as RSE5b. Consequently, a chromosome consisting of the short arm of RSE6a and the long arm of RSE5a will be called RSE6b. Of the additional 4 specimens of RSE studied, only one showed the heterozygous condition as found in female No. 325 (table 1). The other 3 specimens were homozygous for RSE5b and RSE6b. A G-band comparison of the chromosomes involved in the WART is depicted in figure 3 . To confirm the assignment of chromosomal arms and the assumption of a WART, FISH experiments were conducted (see below).
In addition to centromeric heterochromatin, CBGbanding revealed large pericentromeric blocks on 6 autosomal pairs and the $\mathrm{X}$, and a terminally located heterochromatic band on the short arm of RSE8 (fig. 4). Size variation of these heterochromatic segments was observed intra- and interindividually. The autosomal pericentromeric heterochromatin blocks were quinacrineand DAPI-negative but brightly stained by CMA. In contrast, the heterochromatin of the $\mathrm{X}$ chromosome, although also being CMA-positive, showed a moderate fluorescence with quinacrine and DAPI. Compared to other Rhinolophidae species, the karyotype of RSE thus contains quite a large amount of CBG-positive heterochromatin and represents a further example in Chiroptera where the reduction of the diploid number is accompanied by acquisition of heterochromatin [see also Volleth and Heller, 2007].

The secondary constriction on the long arm of RSE9, situated close to the centromere, was shown to be an active NOR by Ag-staining.

\section{Male Sex Chromosome System}

Of the 2 males captured, fibroblast culture yielded poor harvest in one and failed completely in the second specimen. Nevertheless, it became clear that despite a conserved chromosome number, a Y-autosomal translocation must have occurred in this species. In addition to the chromosomes involved in the WART, the karyotype of the male displayed 4 unpaired chromosomes (fig. 5). Besides the $\mathrm{X}$ chromosome $\left(\mathrm{X}_{1}\right)$, there was only one homolog of RSE7 $\left(\mathrm{X}_{2}\right)$. Further, a single medium-sized metacentric chromosome $\left(\mathrm{Y}_{1}\right)$ with a heterochromatic arm (probably representing the true $\mathrm{Y}$ chromosome) and one small subtelocentric chromosome $\left(\mathrm{Y}_{2}\right)$ with a heterochromatic short arm were found. According to the Gbanding pattern, the long arm of $\mathrm{Y}_{2}$ could be homologous 


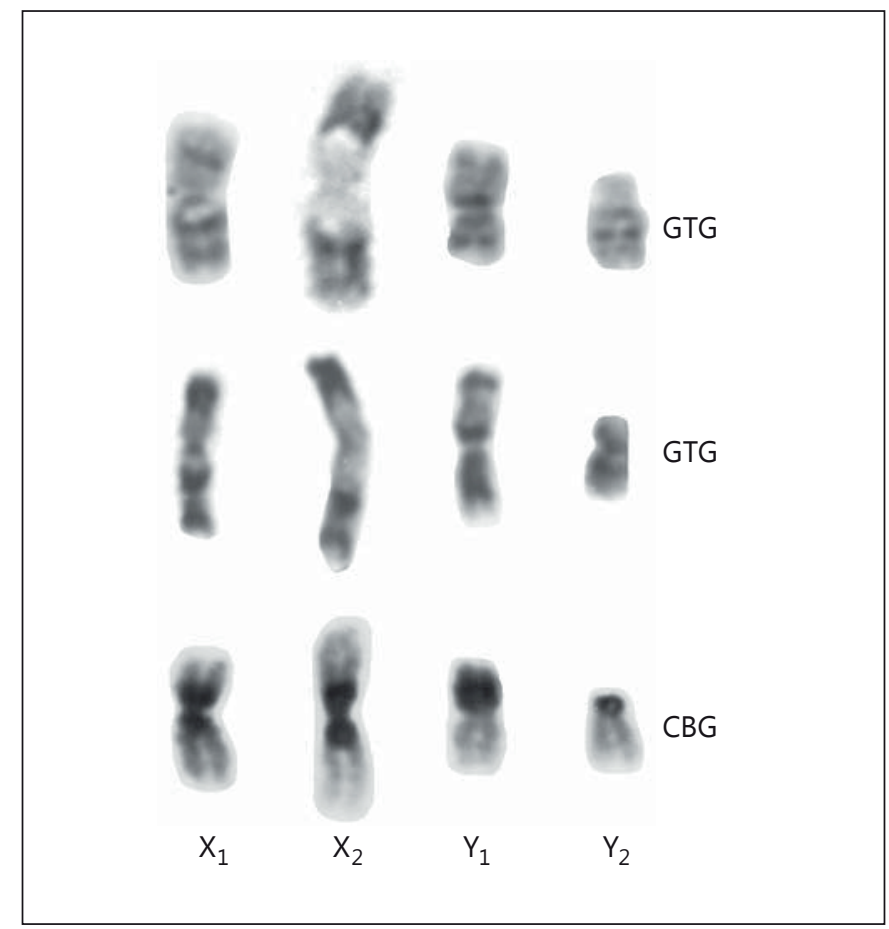

Fig. 5. G-banded (upper and middle row) and C-banded (lower row) gonosomes of RSE male No. 214. Of the 4 unpaired chromosomes, $\mathrm{X}_{1}$ and $\mathrm{X}_{2}$ showed homology to the $\mathrm{X}$ chromosome and to RSE7 of the females, respectively. $Y_{1}$ and $Y_{2}$ were found only in the male.

to the short arm of RSE7. The banding pattern of the euchromatic part of the medium-sized element, $\mathrm{Y}_{1}$, however, seems to be different from that of the long arm of RSE7. Therefore, in addition to the suggested translocation between RSE7 and the ancestral Y, further chromosomal rearrangements have presumably occurred in the sex chromosome system of RSE. Unfortunately, FISH experiments elucidating the proposed $\mathrm{X}_{1} \mathrm{X}_{2} \mathrm{Y}_{1} \mathrm{Y}_{2}$ sex chromosome system could not be performed due to lack of material.

\section{FISH}

The complete set of whole chromosome painting probes from the vespertilionid bat M. myotis [Ao et al., 2006] was used in FISH experiments. The assignment of these probes to chromosomal arms of RSE is shown in figure 1. In summary, 22 MMY probes resulted in 31 painted segments. Painting probes from some acrocentric MMY chromosomes detected 2 homologous segments each in RSE (i.e. MMY7, 10, 12, 20, and 22), and a single probe, MMY8, painted 3 segments. Only 2 chro-

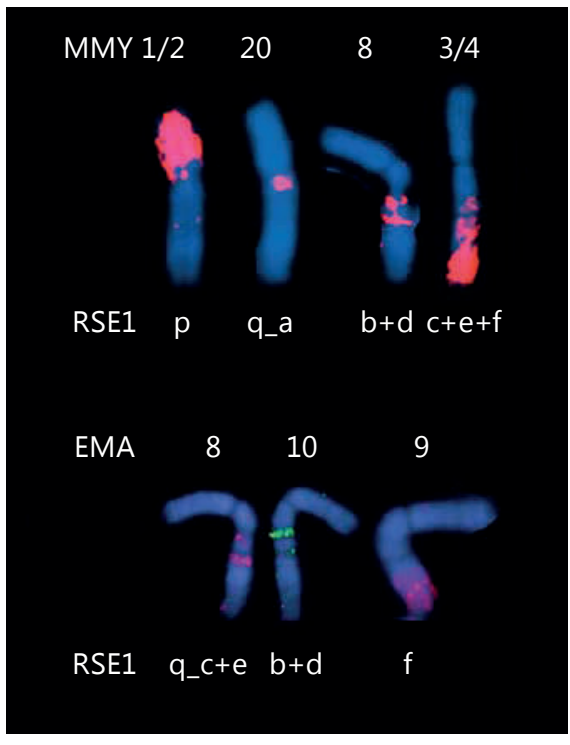

Fig. 6. Segmental composition of RSE1 as revealed by FISH. Using MMY paints (upper row) and EMA paints (lower row), it could be shown that the long arm is composed of tandemly arranged segments homologous to MMY20, 8, and 3 from centromere to telomere. Additionally, RSE1q was shaped by a paracentric inversion. The proximal breakpoint was located within the MMY8i homologous segment. Using the probes EMA8 and EMA9, the distal breakpoint situated in the MMY3 homologous segment could be refined. The proximal part of MMY3 is homologous to HSA6 (detected here by EMA8), the distal part to HSA1 (EMA9). According to the FISH results, the inversion breakpoint is situated within the EMA8 (and, therefore, within the HSA6) homologous segment.

mosomal arms of RSE were shown to be homologous to more than one MMY chromosomal arm, i.e. the long arm of RSE1 and the short arm of RSE3 (fig. 1). In addition to the proposed tandem fusion (see discussion), a paracentric inversion, comprising the segments homologous to MMY3 and MMY8, has further shaped the long arm of RSE1 (fig. 6).

According to the FISH results using MMY probes, the short and the long arm of RSE5a were homologous to MMY5 and MMY4, respectively. Since this arm combination was also found in the family Hipposideridae (see Discussion), we consider chromosome forms RSE5a and RSE6a as the plesiomorphic condition. Consequently, the homozygous occurrence of RSE5b and RSE6b is considered as the derived state. RSE6a showed homology to MMY14 in the short arm and to MMY9 in the long arm. According to the presumed WART, RSE5b should be composed of MMY5 and MMY9 and RSE6b of MMY14 and MMY4. This hypothesis was confirmed by 2 -color 


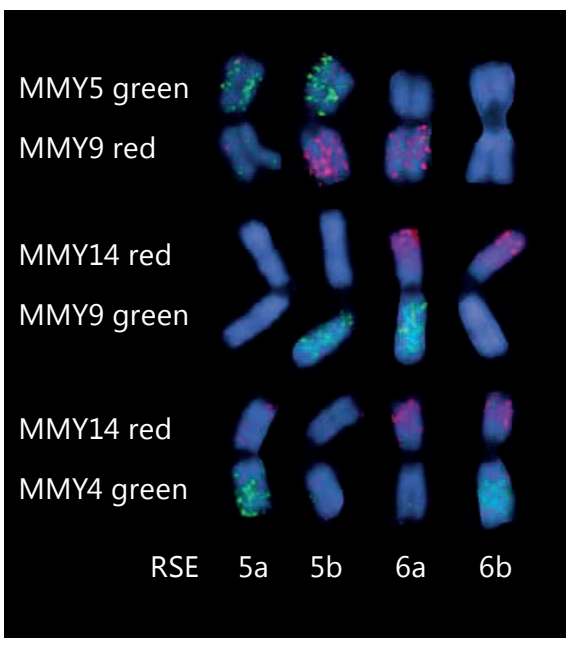

Fig. 7. Dual-color hybridization with MMY chromosome-specific painting probes showing the heterozygous state $(5 a, 5 b, 6 a, 6 b)$ for the chromosomes involved in the WART. In each of the 3 experiments shown, one chromosome is highlighted by both probes, 2 were stained by only one probe and one chromosome remained without any hybridization signal. The MMY probes used are indicated at the left side.

FISH experiments in the heterozygous female No. 325 (fig. 7). For example, co-hybridization of the digoxigeninlabeled probe MMY14, detected by Cy3 antibodies, and the biotinylated probe MMY9, visualized by Avidin Alexa Fluor 488, resulted in one chromosome showing red signals in the short arm and green signals in the long arm (i.e. RSE6a), one chromosome displaying only red signals (i.e. RSE6b), one chromosome with only green signals (i.e. RSE5b), and one chromosome without any hybridization signals (i.e. RSE5a). The combination of probes MMY3/4 and MMY14 and of probes MMY5/6 and MMY9 gave respective results (fig. 7). Therefore, the heterozygous state resulting from a WART between RSE5a and RSE6a was confirmed in female No. 325, the mother of the fetus displaying the ancestral homozygous condition RSE5a and RSE6a.

The NOR-bearing chromosomal arm RSE9q was proven by FISH to be homologous to MMY21. This location was proposed as a synapomorphic character for the families Rhinolophidae and Hipposideridae, as the MMY21 homologous chromosomal arm displayed a secondary constriction in all species investigated so far [Volleth et al., 2002; Volleth and Eick, 2012].

To supplement the results obtained with MMY probes, a selected panel of probes from the tree shrew (T. belangeri, TBE) [Müller et al., 1999] and a lemur (E. macaco,
EMA) [Müller et al., 1997] was used for FISH. These experiments enabled (a) differentiation between the short and long arms of the MMY bi-armed pairs and (b) discrimination in cases where 2 segments in RSE showed homology to the same MMY chromosome. Table 2 gives an overview of the probes used, the results obtained and the interpretation concerning the homology to Myotis and human.

\section{Discussion}

\section{Chromosomal Evolution in Rhinolophidae}

The diploid number of $2 n=28$ found in RSE is the lowest reported for any taxon of the horseshoe bat family. The highest chromosome number reported for Rhinolophidae, $2 \mathrm{n}=62$, is found in the majority of Asian species. This $2 \mathrm{n}=62$ karyotype is composed of acrocentric autosomal elements only in all 13 species studied up to now. G-band comparison and painting with bat-specific probes confirmed the presumed karyotype conservation in these cases [Mao et al., 2007]. The FNa in the $2 \mathrm{n}=62$ all-acrocentric karyotype as well as in most of the remaining $R h i-$ nolophus species is 60 (or, if a tiny element is considered as bi-armed, $\mathrm{FNa}=62$, e.g. in $R$. ferrumequinum nippon [Ando and Uchida, 1974; see also Volleth et al., 2013]). Conservation of entire chromosomal arms during rhinolophid karyotype evolution was confirmed in all species studied by banding and FISH techniques [Mao et al., 2007; Volleth et al., 2013]. Consequently, Robertsonian translocations have been proposed as the predominant mode of chromosomal evolution in Rhinolophidae [Mao et al., 2007].

A karyotype consisting of only bi-armed elements with 60 autosomal arms would show a diploid number of 32 (30 autosomes plus 2 gonosomes). For acquisition of a lower $2 \mathrm{n}$, other modes of rearrangements in addition to Robertsonian translocations are necessary. In RSE, the reduction of the diploid number to $2 \mathrm{n}=28$ and $\mathrm{FNa}=52$ was very likely obtained by tandem fusions. Accordingly, chromosomal arms RSE1q and RSE3p each were painted by 3 different Myotis chromosomes which have previously been shown to be homologous to entire chromosomal arms in rhinolophid karyotypes [Ao et al., 2007; Volleth et al., 2013].

Concerning the diploid chromosome number, the family Rhinolophidae can roughly be divided into 3 groups, 2 of which are characterized by karyotype conservatism [reviewed in Zima et al., 1992]. The first group comprises the so-called 'African clade' with a $2 n=58$ 
Table 2. Results of FISH experiments with EMA and TBE probes on RSE chromosomes

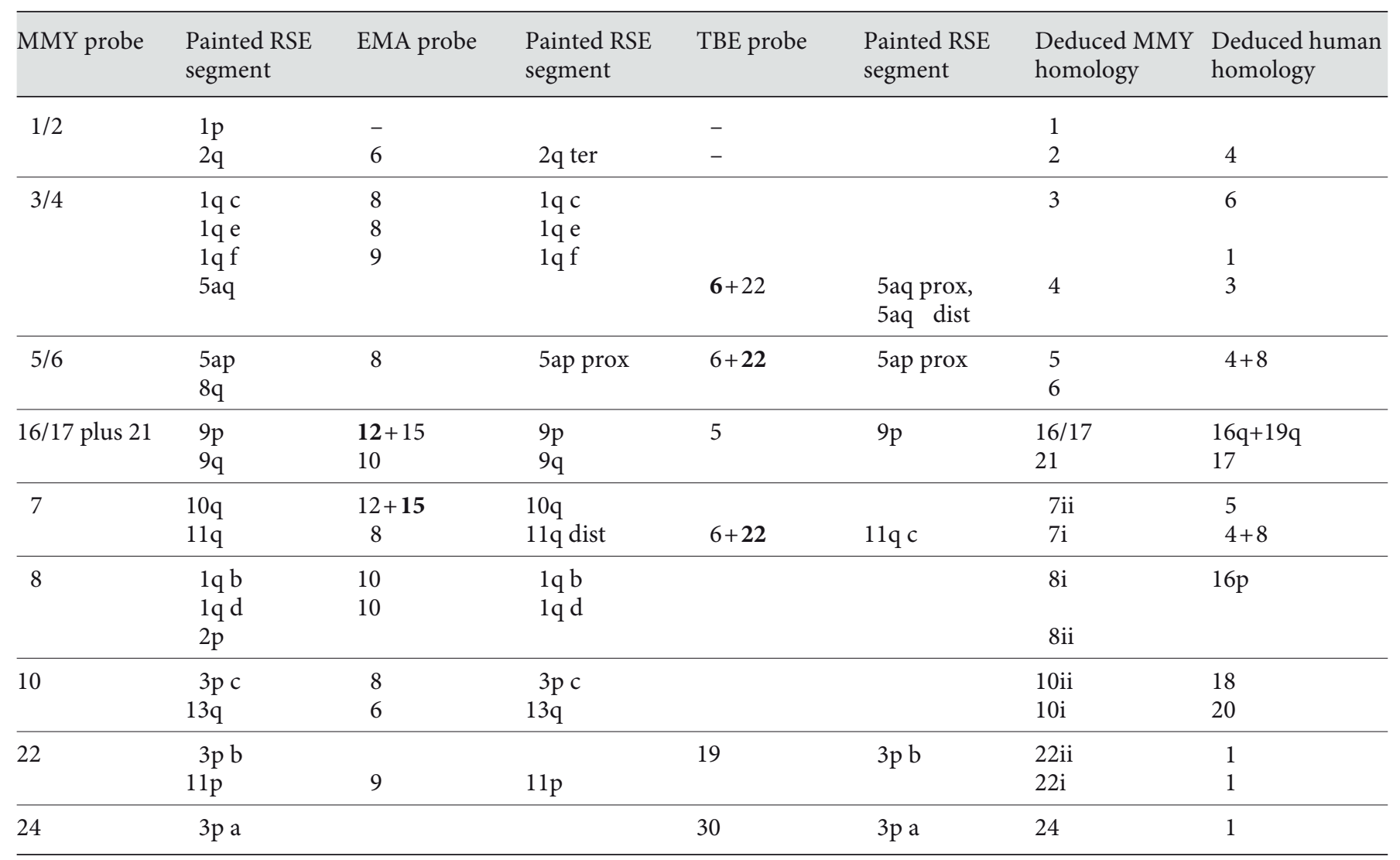

Painting probes from EMA and TBE were used to differentiate between segments showing homology to the same MMY probe except for segments homologous to MMY12 and MMY20, which are represented as one entity also in EMA and TBE. Homology of MMY chromosomal segments to EMA and TBE probes was described in Volleth et al. [2011]. For the cases where a sorting peak contained 2 chromosomes, the chromosome homologous to the respective MMY chromosome is indicated in bold type.

$\mathrm{a}-\mathrm{f}=$ Segments within a chromosomal arm from centromere to telomere. Indicated are only those segments painted by a respective EMA or TBE probe which are related to the MMY probe in question. dist $=$ Distal; prox $=$ proximal. karyotype, inhabiting mainly Africa and Europe (16 species studied so far), plus $R$. hipposideros $(2 \mathrm{n}=54,56,58)$ [see Volleth et al., 2013]. The second group consists of 13 Asian species with $2 n=62$. The third, diverse group with chromosome numbers ranging from 28 to 60 contains 14 Asian species belonging to different phylogenetic lineages. However, only 6 species of the third group have been studied by G-banding and FISH [Zima et al., 1992; Mao et al., 2007; this study; own unpubl. results]. Karyotype comparisons revealed that only few chromosomal arm combinations are shared among these species. This fact points to extensive chromosomal arm exchange during chromosomal evolution in this group.

With exception of 2 ancestral, small metacentric elements present in the first group (i.e. the African clade and
R. hipposideros), the only metacentric autosome observed in more than 2 Rhinolophus species is composed of chromosomal arms homologous to M. myotis MMY4 and MMY5 (i.e. human homology HSA3-21 and HSA13-8-4). This MMY4/5 combination occurs in RSE, $R$. pearsoni pearsoni [Mao et al. 2007] and R. hipposideros [Volleth et al., 2013]. Although convergent evolution can never be excluded in such cases, the presence of this arm combination in the closely related family Hipposideridae (Hipposideros armiger, $H$. larvatus, $H$. pomona, $H$. pratti) [Volleth et al., 2002; Mao et al., 2007, 2010] points to a possible common origin. We, therefore, consider this arm combination, MMY4/5, to be an ancestral element in the rhinolophid karyotype. Consequently, RSE chromosomes composed of elements homologous to MMY4/5 and 
MMY9/14 are thought to represent the ancestral, and after WART, MMY4/14 and MMY5/9 the derived condition.

\section{Y-Autosome Translocation}

Gonosome-autosome translocations have been found in several mammalian orders, but they are, nevertheless, rare exceptions from the normal XY condition [Ashley, 2002]. Cases where an autosome is translocated either to the $\mathrm{X}$ or to the $\mathrm{Y}$ chromosome are easily detected by the resulting odd diploid chromosome number. In RSE, however, both sexes show the same diploid chromosome number. After application of different banding techniques, it became obvious that the second homolog of RSE7 was missing in the male. We, therefore, propose a $\mathrm{X}_{1} \mathrm{X}_{2} \mathrm{Y}_{1} \mathrm{Y}_{2}$ sex chromosome system based on a translocation between an autosome, RSE7, and the former Y chromosome. Surprisingly, in another species of the $R$. trifoliatus group, living in the same habitat as RSE, a different autosome is involved in a $\mathrm{Y}$-autosomal translocation [Volleth et al., in preparation]. Detailed description of such rearrangements can only be achieved by application of partial paints or other FISH probes which differentiate the chromosomal segments involved in the Y-autosome rearrangement. In the case of Alouatta species (Platyrrhini, Primates), such experiments showed that the underlying chromosomal rearrangement leading to the $\mathrm{X}_{1} \mathrm{X}_{2} \mathrm{Y}_{1} \mathrm{Y}_{2}$ system is not a simple WART, but that $\mathrm{Y}_{1}$ harbors segments homologous to both arms of the former autosome, $\mathrm{X}_{2}$ [de Oliveira et al., 2002]. Although FISH experiments could not be done in RSE, G-band analysis points to the fact that a complex rearrangement is likely to have shaped the Y-autosomal elements in this species.

\section{WART}

The whole-arm translocation between 2 nonhomologous bi-armed chromosomes (type-a WART) [Hauffe and Pialek, 1997; White et al., 2010] observed in the horseshoe bat species RSE represents one of the rare documented cases of WARTs in mammals so far. In the last years, WARTs have been proposed as a mechanism for chromosome evolution in different mammalian taxa [Hauffe and Pialek, 1997; Britton-Davidian et al., 2005; Veyrunes et al., 2007; White et al., 2010]. However, nearly all specimens displaying monobrachial homology in bi-armed chromosomes were found to be hybrids between 2 chromosomal races. The formation of a new chromosomal arm combination by a WART, however, was detected very rarely and only in single specimens, e.g. Mus [Capanna and Redi, 1995; Castiglia and Capanna,
1999; Catalan et al., 2000; Veyrunes et al., 2007], Sorex [Pavlova et al., 2008; Fedyk and Chetnicki, 2009] and Tupaia [Hirai et al., 2002]. Our observation in RSE is the second case where a WART is found as polymorphism in several individuals, which cannot be assigned to an obvious hybrid zone between different chromosomal races. Hence, it can be speculated that this WART may be involved in the chromosomal evolution of this species. The other example concerns the agile gibbons from Sumatra and Thailand [Van Tuinen et al., 1999; Hirai et al., 2003, 2005; Tanomtong et al., 2010]. In H. agilis, the majority of specimens examined showed the homozygous derived constellation of the WART, and only few specimens from Sumatra were proven to be heterozygous. The homozygous ancestral state, however, has not been found so far [Hirai et al., 2005]. In contrast, all 3 constellations connected with the WART were found among the 6 analyzed specimens of the horseshoe bat RSE presented in our study (table 1). The 4 chromosomal arms involved in the WART in RSE were shown to be homologous to MMY chromosomes 4, 5, 9, and 14. The arm combination MMY4/5 is considered as an ancestral element of Rhinolophidae (see above). Therefore, the presence of the biarmed chromosomes MMY4/5 and MMY9/14 is thought to be the ancestral condition. The arm combination MMY4/14 and MMY5/9, resulting from the WART, would then represent a derived state.

Phenetic analysis of morphological characters resulted in the placement of RSE together with R. luctus in the trifoliatus group, which is characterized by its unique noseleaf morphology [Bogdanowicz, 1992; Csorba et al., 2003]. Molecular studies of cytochrome b sequences confirmed the close relationships of these 3 species [GuillénServent et al., 2003; Sazali et al., 2011]. Other members of this group show diploid chromosome numbers ranging from $2 \mathrm{n}=52$ ( $R$. formosae) [Ando et al., 1983] to $2 \mathrm{n}=32$ (R. luctus, R. beddomei) [Naidu and Gururaj, 1984; Harada et al., 1985; Hood et al., 1988; Koubínová et al., 2010]. However, no arm combination was found to be shared between RSE and the closely related members of the trifoliatus group living in the same habitat, i.e. R. luctus and $R$. trifoliatus, both with a diploid number of $2 \mathrm{n}=32$ [Volleth et al., in preparation]. This fact mirrors the rapid rate of chromosomal evolution in this group. If we assume an all-metacentric karyotype with 32 chromosomes also for the ancestor of the trifoliatus group, the only mode of rearrangement which could change arm combinations in a single step is WART. It is a remarkable parallelism that in the Hylobatidae, one of the mammalian taxa with the highest rate of chromosomal rearrangements [Capozzi et 
al., 2012], the karyotypes of 2 sibling species, i.e. H. agilis from Sumatra and H. albibarbis from Borneo (a former subspecies of $H$. agilis), also differ by a WART [Hirai et al., 2005]. The presence of WART heterozygotes in $H$. agilis was interpreted as an intermediate stage in the differentiation of the karyotype [Van Tuinen et al., 1999].

In contrast to mice and shrews, chromosomal races seem to be extremely rare in bats. The only known example is Uroderma bilobatum, a phyllostomid bat species (or species complex) presenting with different diploid numbers $(2 \mathrm{n}=38,42,44)$. Secondary contact of the $2 \mathrm{n}=$ 38 and $2 n=44$ races resulted in a narrow hybrid zone with intermediate diploid numbers (39-41) [Owen and Baker, 2001, and citations therein; Hoffmann et al., 2003].

Our study is the first dealing with karyology in RSE, a species without described subspecies and only distributed in western Malaysia and Borneo. The existence of a hy- brid zone seems to be rather improbable. In our opinion, it is much more likely that the WART observed in RSE mirrors ongoing chromosomal evolution within this species. However, whether this WART is a simple polymorphism, representing the last step prior to fixation of the RSE karyotype, or whether it is a step towards the derived karyotype of a new (sub)species will probably never be resolved. Sadly, it is much more likely that this species, adapted to a life in the interior of tropical rainforests [Kingston, 2013], will lastly disappear from our planet due to man-made destruction of its habitat.

\section{Acknowledgements}

For support on field expeditions in 1984 and 1992, we cordially thank all our friends and colleagues who accompanied us. H.-S.Y. was supported by MoHE-HIR grant H-50001-00-A000025.

\section{References}

Ando K, Uchida TA: Karyotype analysis in Chi- Capanna E, Redi CA: Whole-arm reciprocal roptera II. Phylogenetic relationships in the genus Rhinolophus. Sci Bull Fac Agr, Kyushu Univ 28:119-129 (1974).

Ando K, Yasuzumi F, Tagawa T, Uchida TA: Further study on the karyotypic evolution in the genus Rhinolophus (Mammalia: Chiroptera). Caryologia 46:101-111 (1983).

Ao L, Gu X, Feng Q, Wang J, O’Brien PC, et al: Karyotype relationships of six bat species (Chiroptera, Vespertilionidae) from China revealed by chromosome painting and Gbanding comparison. Cytogenet Genome Res 115:145-153 (2006).

Ao L, Mao X, Nie W, Gu X, Feng Q, et al: Karyotypic evolution and phylogenetic relationships in the order Chiroptera as revealed by G-banding comparison and chromosome painting. Chromosome Res 15:257-267 (2007).

Ashley T: X-Autosome translocations, meiotic synapsis, chromosome evolution and speciation. Cytogenet Genome Res 96:33-39 (2002).

Baker RJ, Bickham JW: Karyotypic evolution in bats: evidence of extensive and conservative chromosomal evolution in closely related taxa. Syst Zool 29:239-253 (1980).

Bogdanowicz W: Phenetic relationships among bats of the family Rhinolophidae. Acta Theriol 37:213-240 (1992).

-Britton-Davidian J, Catalan J, da Garça Ramalhinho M, Auffray JC, Nunes AC, et al: Chromosomal phylogeny of Robertsonian races of the house mouse on the island of Madeira: testing between alternative mutational processes. Genet Res 86:171-183 (2005). translocation (WART) between Robertsonian chromosomes: finding of a Robertsonian heterozygous mouse with karyotype derived through WARTs. Chromosome Res 3:135137 (1995).

Capozzi O, Carbone L, Stanyon RR, Marra A, Yang F, et al: A comprehensive molecular cytogenetic analysis of chromosome rearrangements in gibbons. Genome Res 22:2520-2528 (2012).

Castiglia R, Capanna E: Whole-arm reciprocal translocation (WART) in a feral population of mice. Chromosome Res 7:493-495 (1999).

Catalan J, Auffray JC, Pellestor F, Britton-Davidian J: Spontaneous occurrence of a Robertsonian fusion involving chromosome 19 by single whole-arm reciprocal translocation (WART) in wild-derived house mice. Chromosome Res 8:593-601 (2000).

Csorba G, Ujhelyi P, Thomas N: Horseshoe Bats of the World (Chiroptera: Rhinolophidae) (Alana Books, Bishop's Castle 2003).

$\checkmark$ de Oliveira EHC, Neusser M, Figueiredo WB, Nagamachi C, Pieczarka JC, et al: The phylogeny of the howler monkeys (Alouatta, Platyrrhini): reconstruction by multicolor crossspecies chromosome painting. Chromosome Res 10:669-683 (2002).

Fedyk S, Chetnicki W: Whole-arm reciprocal translocation in a hybrid population of Sorex araneus. Chromosome Res 17:451-454 (2009).

Fredga K: The chromosome races of Sorex araneus in Scandinavia. Hereditas 125:123-135 (1996).
Guillén-Servent A, Francis CM, Ricklefs RE: Phylogeny and biogeography of the horseshoe bats, in Csorba G, Ujhelyi P, Thomas N (eds): Horseshoe Bats of the World (Chiroptera: Rhinolophidae), pp xii-xxiv (Alana Books, Bishop's Castle 2003).

Halkka L, Söderlund V, Skaren U, Heikkila J: Chromosomal polymorphism and racial evolution of Sorex araneus L. in Finland. Hereditas 106:257-275 (1987).

Harada M, Yenbutra S, Yosida TH, Takada S: Cytogenetical study of Rhinolophus bats (Chiroptera, Mammalia) from Thailand. Proc Japan Acad Ser B 61:455-458 (1985).

Hauffe HC, Pialek J: Evolution of the chromosomal races of Mus musculus domesticus in the Rhaetian Alps: the roles of whole-arm reciprocal translocation and zonal raciation. Biol J Linn Soc 62:255-278 (1997).

Heller KG, Achmann R, Witt K: Monogamy in the bat Rhinolophus sedulus? Z Säugetierkd 58:376-377 (1993).

Hirai H, Hirai Y, Kawamoto Y, Endo H, Kimura J, Rerkamnuaychoke W: Cytogenetic differentiation of two sympatric tree shrew taxa found in the southern part of the Isthmus of Kra. Chromosome Res 10:313-327 (2002).

-Hirai H, Mootnick AR, Takenaka O, Suryobroto B, Mouri T, et al: Genetic mechanism and property of a whole-arm translocation (WAT) between chromosomes 8 and 9 of the agile gibbons (Hylobates agilis). Chromosome Res 11:37-50 (2003).

-Hirai H, Wijayanto H, Tanaka H, Mootnick AR, Hayano A, et al: A whole-arm translocation (WAT8/9) separating Sumatran and Bornean agile gibbons, and its evolutionary features. Chromosome Res 13:123-133 (2005). 
-Hoffmann FG, Owen JG, Baker RJ: mtDNA perspective of chromosomal diversification and hybridization in Peters' tent-making bat (Uroderma bilobatum: Phyllostomidae). Mol Ecol 12:2981-2993 (2003).

Hood CS, Schlitter DA, Georgudaki JI, Yenbutra S, Baker RJ: Chromosomal studies of bats (Mammalia: Chiroptera) from Thailand. Ann Carnegie Mus 57:99-109 (1988).

Kingston T: Response of bat diversity to forest disturbance in Southeast Asia: insights from long-term research in Malaysia, in Adams RA, Pedersen SC (eds): Bat Evolution, Ecology, and Conservation, pp 169-185 (Springer, New York 2013).

Koubínová D, Sreepada KS, Koubek P, Zima J: Karyotypic variation in rhinolophid and hipposiderid bats (Chiroptera: Rhinolophidae, Hipposideridae). Acta Chiropterol 12:393400 (2010).

Mao X, Nie W, Wang J, Su W, Ao L, et al: Karyotype evolution in Rhinolophus bats (Rhinolophidae, Chiroptera) illuminated by cross-species chromosome painting and G-banding comparison. Chromosome Res 15:835-848 (2007).

-Mao X, Nie W, Wang J, Su W, Feng Q, et al: Comparative cytogenetics of bats (Chiroptera): the prevalence of Robertsonian translocations limits the power of chromosomal characters in resolving interfamily phylogenetic relationships. Chromosome Res 16:155-170 (2008).

Mao XG, Wang JH, Su WT, Wang YX, Yang FT, Nie WH: Karyotypic evolution in family Hipposideridae (Chiroptera, Mammalia) revealed by comparative chromosome painting, G- and C-banding. Dongwuxue Yanjiu 31: 453-460 (2010).

- Müller S, O'Brien PCM, Ferguson-Smith MA, Wienberg J: Reciprocal chromosome painting between human and prosimians (Eulemur macaco macaco and E. fulvus mayottensis). Cytogenet Cell Genet 78:260-271 (1997).

-Müller S, Stanyon R, O'Brien PCM, FergusonSmith MA, Plesker R, Wienberg J: Defining the ancestral karyotype of all primates by multidirectional painting between tree shrews, lemurs and humans. Chromosoma 108:393-400 (1999).
Naidu KN, Gururaj ME: Karyotype of Rhinolophus luctus (Order: Chiroptera). Curr Sci 53 : 825-826 (1984).

Narain Y, Fredga K: A hybrid zone between the Hällefors and Uppsala chromosome races of Sorex araneus in central Sweden. Hereditas 125:137-145 (1996).

Owen JG, Baker RJ: The Uroderma bilobatum (Chiroptera: Phyllostomidae) cline revisited. J Mammalogy 82:1102-1113 (2001).

Pavlova SV, Kolomiets OL, Bulatova NS, Searle JB: Demonstration of a WART in a hybrid zone of the common shrew (Sorex araneus Linnaeus, 1758). Comp Cytogenet 2:115-120 (2008).

Sazali SN, Besar K, Abdullah MT: Phylogenetic analysis of the Malaysian Rhinolophus and Hipposideros inferred from partial mitochondrial DNA cytochrome b gene sequences. Pertanika J Trop Agric Sci 34:281-294 (2011).

Simmons NB: Order Chiroptera, in Wilson DE, Reeder DM (eds): Mammal Species of the World. A Taxonomic and Geographic Reference, vol 1, ed 3, pp 312-529 (Johns Hopkins University Press, Baltimore 2005).

Tanomtong A, Khunsook S, Supanuam P, Kaewsri S, Srisamoot N: A discovery of polymorphism of nuclear organizer regions (NORs) and whole-arm translocation (WAT) between chromosome 8 and 9 of lowland agile gibbon (Hylobates agilis unko) in Thailand. Cytologia 75:15-21 (2010)

Van Tuinen P, Mootnick AR, Kingswood SC, Hale DW, Kumamoto AR: Complex, compound inversion/translocation polymorphism in an ape: presumptive intermediate stage in the karyotypic evolution of the Agile Gibbon Hylobates agilis. Amer J Phyl Anthropol 110:129-142 (1999).

Veyrunes F, Watson J, Robinson TJ, BrittonDavidian J: Accumulation of rare sex chromosome rearrangements in the African pygmy mouse, Mus (Nannomys) minutoides: a whole-arm reciprocal translocation (WART) involving an $\mathrm{X}$-autosome fusion. Chromosome Res 15:223-230 (2007).
Veyrunes F, Catalan J, Tatard C, Cellier-Holzem E, Watson J, et al: Mitochondrial and chromosomal insights into karyotypic evolution of the pygmy mouse, Mus minutoides, in South Africa. Chromosome Res 18:563-574 (2010).

Volleth M, Eick G: Chromosome evolution in bats as revealed by FISH: the ongoing search for the ancestral chiropteran karyotype. Cytogenet Genome Res 137:165-173 (2012).

Volleth M, Heller KG: Chromosome number reduction accompanied by extensive heterochromatin addition in the bat Glauconycteris beatrix (Mammalia; Chiroptera, Vespertilionidae). Cytogenet Genome Res 119:245247 (2007).

Volleth M, Heller KG, Pfeiffer RA, Hameister H: A comparative ZOO-FISH analysis in bats elucidates the phylogenetic relationships between Megachiroptera and five Microchiropterian families. Chromosome Res 10:477-497 (2002).

-Volleth M, Yang F, Müller S: High-resolution chromosome painting reveals the first genetic signature for the chiropteran suborder Pteropodiformes (Mammalia: Chiroptera). Chromosome Res 19:507-519 (2011)

Volleth M, Biedermann M, Schorcht W, Heller KG: Evidence for two karyotypic variants of the lesser horseshoe bat (Rhinolophus hipposideros, Chiroptera, Mammalia) in Central Europe. Cytogenet Genome Res 140:55-61 (2013)

White TA, Bordewich M, Searle JB: A network approach to study karyotypic evolution: the chromosomal races of the common shrew (Sorex araneus) and house mouse (Mus musculus) as model systems. Syst Biol 59:262-276 (2010).

Winking H: Some aspects of Robertsonian karyotypic variation in the European wild mice. Current Top Microbiol Immunol 127:68-74 (1986).

Zima J, Volleth M, Horáček I, Cerveny J, Cervena A, et al: Comparative karyology of rhinolophid bats, in Horáček I, Vorhalik V (eds): Prague Studies in Mammalogy, pp 229-236 (Charles University Press, Prague 1992). 